\title{
Tumor del estroma gastrointestinal (G IST) en una paciente con neurofibromatosis tipo 1
}

\author{
Marcelo A Beltrán ${ }^{1,2}$, Carlos Barría ${ }^{3}$, Mario A Contrerasa, \\ Christian S Wilson ${ }^{\mathrm{a}}$, Karina S Cruces $^{1 \mathrm{~b}}$. \\ Gastrointestinal stromal tumor (GIST) \\ in a patient with neurofibromatosis \\ type 1. Report of one case
}

Neurofibromatosis Type 1 (NF1) is an autosomic dominant condition affecting the central nervous systema and presenting a disposition towards development of gastrointestinal stromal tumors (GIST). We report a 38 year-old female patient with neurofibromatosis type 1 that required emergency surgery due to a perforated GIST originating in the fourth duodenal portion. The GIST, and the fourth duodenal portion, were excised and a primary duodenum-jejunal anastomosis was performed. The pathological study showed a partially necrotic solid-cystic tumor with 1 to 2 mitoses per 50-high-power fields. The cells stained positively to CD117, CD34 and Desmin, and were negative to S100, Vimentin, and Smooth Muscle $\alpha$-Actin. The patient is currently asymptomatic and under follow-up during the last 11 months after surgery (Rev Méd Chile 2009; 137: 1197-200).

(Key words: Gastrointestinal stromal tumors; Neurofibromatosis 1; Protein kinase inhitors)
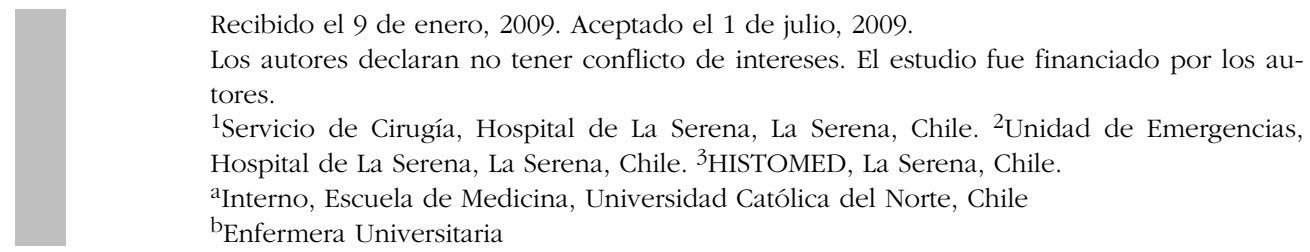

$\mathrm{L}$ a neurofibromatosis tipo 1 (NF1), o enfermedad de von Recklinghausen, es una condición autosómica dominante que afecta al sistema nervioso central. Actualmente se le reconoce como el defecto de un solo gen que compromete múltiples órganos y sistemas, entre ellos el sistema gastrointestinal, y que tiene una particular predisposición hacia la formación de tumores específicos, muchas

Correspondencia a: Dr. Marcelo A Beltrán. Manuel Antonio Caro 2629, Casilla 912, La Serena, IV Región, Chile. E mail: beltran_01@yahoo.com veces malignos ${ }^{1,2}$. Los tumores cutáneos característicos de la NF1, se presentan como neurofibromas discretos, pequeños y benignos en la dermis de más de $95 \%$ de los pacientes, y como neurofibromas plexiformes que afectan porciones de los nervios periféricos e infiltran los tejidos circundantes y tienen el potencial de desarrollar tumores malignos de la vaina nerviosa periférica entre $2 \%$ y $16 \%$ de los $\operatorname{casos}^{1-3}$. Otros tumores que pueden producirse en pacientes con NF1 son los gliomas de la vía óptica, astrocitomas, feocromocitomas, leucemia mieloide juvenil crónica y los tumores del estroma gastrointestinal (GIST) ${ }^{1,3,4}$. Los GIST 
en pacientes con NF1, a diferencia de los GIST en pacientes sin NF1, se desarrollan principalmente en el intestino delgado, son múltiples y pueden asociarse a otros tumores digestivos ${ }^{3,4}$. El riesgo de los pacientes con NF1 de desarrollar un GIST es elevado (7\%). El mecanismo molecular por el cual se producen estos tumores en pacientes con NF1 no ha sido completamente esclarecido pero es diferente al de los GIST esporádicos que se producen en pacientes sin NF1, debido a que implica no sólo mutaciones del gen KIT (c-kit) y del PDGFRA, sino también la inactivación de la neurofibromina y la recombinación mitótica que resulta en la reducción de la homozigosis y pérdida de la heterozigosis de una línea celular germinal, lo que en ausencia de mutaciones del c-kit y del PDGFRA constituye un mecanismo alternativo para la formación de los GISTs ${ }^{4,5}$. Las implicancias de la NF1 para el cirujano han sido ampliamente discutidas ${ }^{6}$, sin embargo la asociación de los GIST con NF1 ${ }^{7-9}$ y con otros tumores intraabdominales como los feocromocitomas $^{10,11}$, constituyen un problema recientemente descrito.

\section{Caso cLínico}

Mujer de 38 años con historia de NF1 que se manifestó en la infancia con abundantes efélides en todo el cuerpo, región axilar e inguinal y neurofibromas discretos. La paciente se presentó en la Unidad de Emergencia del Hospital de La Serena con un cuadro clínico de 7 días de evolución con intenso dolor abdominal, inicialmente epigástrico y posteriormente difuso. Ingresó en shock séptico y mala condición general. Después de estabilización hemodinámica se decidió su intervención quirúrgica con el diagnóstico de abdomen agudo de origen no precisado y peritonitis secundaria. No se realizó ningún estudio radiológico debido a que nuestro hospital no cuenta con radiólogo de turno y tampoco ecografía o tomografía de urgencia. El abordaje del abdomen se realizó a través de una laparotomía mediana. Se aspiraron 3 litros de exudado purulento y en la exploración se evidenció un tumor quístico de $15 \mathrm{~cm}$ de diámetro que se originaba en la $4^{\mathrm{a}}$ porción del duodeno, perforado, adherido al colon sigmoides y cubierto de epiplón mayor (Figura 1). El tumor fue resecado en bloque con el epiplón y la 4a porción del duodeno. El tránsito intestinal se reconstituyó mediante anastomosis término-terminal entre la $3^{\mathrm{a}}$ porción del duodeno y el yeyuno. La evolución postoperatoria fue tórpida, con complicaciones: infección del sitio quirúrgico superficial, íleo intestinal prolongado y neumonía nosocomial. Después de 37 días de hospitalización fue dada de alta en buenas condiciones. El estudio histológico demostró un tumor sólido-quístico, parcialmente necrótico y con bajo índice mitótico (1 a 2 mitosis por 50 campos de aumento mayor). Se observaron células epiteloides (Figura 1A) y fusiformes (Figura 1B), positivas para CD117 (Figura 1C), CD34 y desmina. La reacción inmunohistoquímica fue negativa para $S 100$, vimentina y actina- $\alpha$ de músculo liso. El tamaño del tumor y el cuadro clínico de presentación (necrosis y perforación), constituyen indicaciones para terapia con imatinib por lo que fue derivada al Comité Oncológico Regional para iniciar tratamiento pertinente. La paciente se encuentra asintomática y en seguimiento hace 11 meses.

\section{DisCUSIÓN}

Es sabido que los pacientes con NF1 tienen alto riesgo de desarrollar GISTs ${ }^{4-12}$. La asociación entre GIST y NF1 no es incidental, numerosos estudios genéticos y moleculares han establecido una estrecha relación entre ambas patologías ${ }^{4,5,7}$, sugiriendo su inclusión dentro del espectro clínico de la NF1 $1^{4,11}$. Algunas características de los GIST que se presentan en pacientes con NF1 los hacen sutilmente diferentes de los GIST que se desarrollan en pacientes sin NF1. Entre ellas destaca el predominio del yeyuno e íleon como sitios de desarrollo del tumor y la presencia de múltiples tumores en el asa de intestino comprometida, algunos de los cuales pueden ser benignos y otros malignos $^{7-9}$. Estas características no son frecuentes en GIST que se desarrollan incidentalmente en otros pacientes, los que habitualmente son únicos y predominantemente gástricos ${ }^{8,13-15}$. Una característica de los GIST en pacientes con NF1 es la presentación de múltiples tumores en el íleon ${ }^{8,9}$, lo cual se atribuye al origen del GIST en el plexo mientérico, sin una causa específica claramente establecida ${ }^{8,16}$. Aproximadamente $25 \%$ de los pacientes con NF1 presentan compromiso del sistema gastrointestinal. Los tumores que se desarrollan con mayor frecuencia en estos pacientes son los GIST, siendo sintomáticos entre 5\% y $7 \%$ de los $\operatorname{casos}^{8}$. La presentación clínica de los 


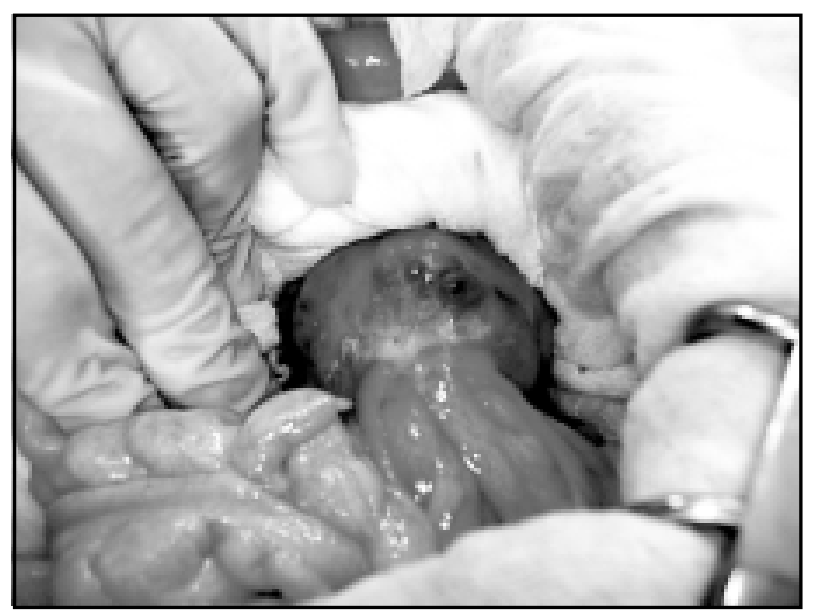

Figura 1. GIST duodenal, necrótico y perforado cubierto de epiplón y pared del colon sigmoides, los GIST quísticos del duodeno son raros.

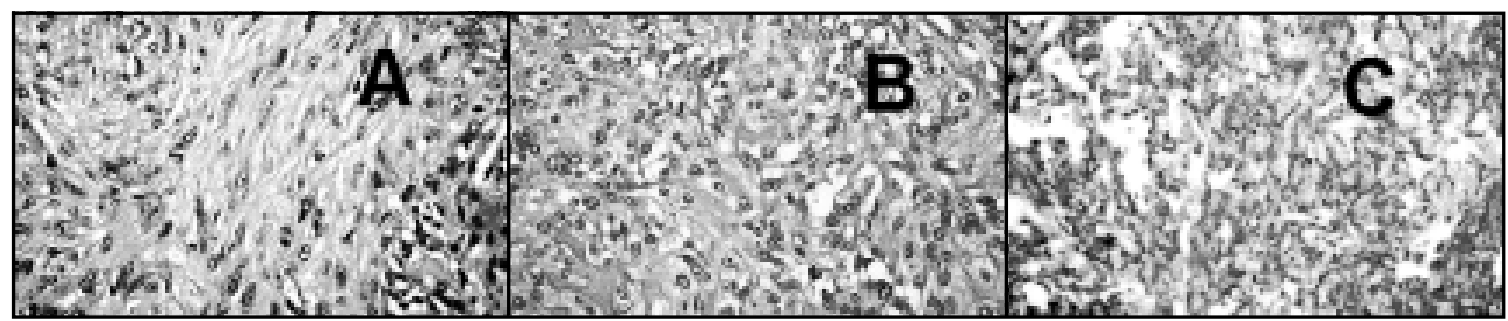

Figura 2. A) Microfotografía en la que se observa algunas áreas del tumor con predominio de células epiteloides (HE 40x). B) En otras áreas el predominio fue de células fusiformes (HE 40x). C) Se observaron áreas del tumor con un patrón celular mixto sin predominio de un tipo celular sobre otro (CD117 40x).

GIST en NF1 es similar a la presentación clínica en otros pacientes: ulceración, hemorragia digestiva, masa palpable, obstrucción intestinal, vólvulo, intususcepción y perforación $8,9,12-15$. Nuestra paciente se presentó con un cuadro de urgencia por un GIST duodenal perforado, la cual constituye una presentación conocida y frecuente de los tumores del intestino delgado independientemente de si son GIST u otro tipo de tumor, como los adenocarcinomas o linfomas intestinales ${ }^{17}$.

En el estudio radiológico de los GIST la tomografía abdominal computarizada demuestra claramente la lesión y permite planificar la estrategia quirúrgica. Otros estudios que pueden ser de utilidad son la ecografía abdominal y la resonancia magnética ${ }^{18}$. En nuestro caso no fue posible realizarlos para planificar la cirugía. Los GIST del duodeno son raros y su tratamiento quirúrgico aún es controversial. Tradicionalmente se propone la pancreatoduodenectomía para el tratamiento de los GIST de la segunda, tercera o cuarta porción del duodeno ${ }^{19}$. Recientemente se han descrito resecciones segmentarias del duodeno como tratamiento efectivo de los GIST duodenales, con buenos resultados ${ }^{19-22}$. En nuestro caso el GIST localizado en la cuarta porción del duodeno se trató mediante resección segmentaria con márgenes negativos y anastomosis primaria entre duodeno y yeyuno.

Debido al tamaño del tumor y a la necrosis y perforación asociada, esta paciente se encuentra en riesgo de recurrencia tumoral por lo que fue derivada para tratamiento con imatinib. La terapia con imatinib se encuentra claramente establecida en GISTs metastáticos, en recurrencias y en casos de perforación tumoral ${ }^{23,24}$, como la que presentó nuestra paciente. Aún es controversial la indicación de imatinib en tumores malignos con riesgo 
elevado de recurrencia. Los GIST son parte del espectro de tumores que los pacientes afectados por NF1 se encuentran en riesgo de desarrollar. Su

\section{REFERENCIAS}

1. Lynch TM, Gutmann DH. Neurofibromatosis 1. Neurol Clin North Am 2002; 20: 841-65.

2. Reynolds RM, Browning GGP, Nawroz I, Campbell IW. Von Recklinghausen's neurofibromatosis: Neurofibromatosis type 1 . Lancet 2003; 361: 1552-4.

3. Radtke HB, Sebold CD, Allison C, Haidle JL, Schneider G. Neurofibromatosis type 1 in genetic counseling practice: Recommendations of the National Society of Genetic Counselors. J Genet Counsel 2007; 16: 387-407.

4. Maertens O, Prenen H, Debiec-Rychter M, Wozniak A, Sciot R, Pauwels P ET al. Molecular pathogenesis of multiple gastrointestinal stromal tumors in NF1 patients. Human Mol Gen 2006; 15: 1015-23.

5. Stewart DR, Corless Cl, Rubin BP, Heinrich MC, Messiaen LM, Kessler LJ et al. Mitotic recombination as evidence of alternative pathogenesis of gastrointestinal stromal tumors in neurofibromatosis type 1 . J Med Genet 2007; 44: e61.

6. Seymour-Dempsey K, Andrassy RJ. Neurofibromatosis: Implications for the general surgeon. J Am Coll Surg 2002; 195: 553-61.

7. Giuly Ja, Pican R, Giuly D, Monges B, Nguyen-Cat R. Von Recklinghausen disease and gastrointestinal stromal tumors. Am J Surg 2003; 185: 86-7.

8. Joo M, Lee HK, Kiм H, Kim MK, Chi JG. Multiple small intestinal stromal tumors associated with neurofibromatosis 1. Yonsei Med J 2004; 45: 564-7.

9. Beltrán ma, Cruces KS, Barría C, Verdugo G. Multiple gastrointestinal stromal tumors of the Ileum and neurofibromatosis type 1. J Gastrointest Surg 2006; 10: 297-301.

10. Teramoto S, Ota T, Maniwa A, Matsui T, itaya N, Aoyagi K ET al. Two von Recklinghausen's disease cases with pheochromocytomas and gastrointestinal stromal tumors (GIST) in combination. Int J Urol 2007; 14: 73-4.

11. Kramer K, Hasel C, Aschoff AJ, Henne-Bruns D, Wuerl P. Multiple gastrointestinal stromal tumors and bilateral pheochromocytoma in neurofibromatosis. World J Gastroenterol 2007; 13: 3384-7.

12. Bórquez PM, Neveu RC. Tumores del estroma gastrointestinal (GIST), un particular tipo de neoplasia. Rev Méd Chile 2008; 136: 921-9. comportamiento biológico, estudio, tratamiento y pronóstico son similares a los GIST que se desarrollan incidentalmente en pacientes sin NF1.

13. Beltrán ma, Pujado B, Pozo C, Méndez P, Barrera R, Mora R. Tumor gástrico gigante del estroma gastrointestinal. Gastroenterol Latinoam 2007; 18: 378-82.

14. Benavides CC, Burmeister RL, Apablaza SP, García CC, Pinedo MV. Tumores gástricos estromales. Rev Chil Cir 2002; 54: 44-8.

15. Braghetto I, Parada FJ, Cardemil G, Csendes A, Fernández E, Korn O et al. Tumores gastrointestinales estromales (GIST): Experiencia del Servicio de Cirugía del Hospital Clínico de la Universidad de Chile entre 1999 y 2005. Rev Méd Chile 2007; 135: 551-7.

16. Walsh NM, Bodurtha A. Auerbach's myenteric plexus: A possible site of origin for gastrointestinal stromal tumors in von Recklinghausen's neurofibromatosis. Arch Pathol Lab Med 1990; 114: 522-5.

17. Beltrán MA, Cruces KS. Primary tumors of Jejunum and Ileum as a cause of intestinal obstruction: A case control Study. Int J Surg 2007; 5: 183-91.

18. Oyanedel RQ, O'brien AS, Pizarro AG, Zamora ER, Menias CO. Tumor estromal gastrointestinal (GIST): Formas de presentación. Rev Chil Radiol 2005; 11: 13-8.

19. Carvajal C, iturra S, Justiniano JC, Bustamante MZ, Contreras JE, Lombardi JS et al. Tumores estromales gastrointestinales duodenales. Rev Méd Chile 2006; 134: 481-4.

20. Asakawa M, Sakamoto Y, Kajiwara T, Nara S, Esaki M, Shimada K ET AL. Simple segmental resection of the second portion of the duodenum for the treatment of gastrointestinal stromal tumors. Langenbecks Arch Surg 2008; 393: 605-9.

21. Liyanage CAH, Abeygunawardhana S, Kumarage S, Deen KI. Duodenum-preserving local excision of a gastrointestinal stromal tumor. Hepatobiliary Pancreat Dis Int 2008; 7: 214-6.

22. Mennigen R, Wolters HH, Schulte B, Pelster FW. Segmental resection of the duodenum for gastrointestinal stromal tumor (GIST). World J Surg Oncol 2008; 6: 105-10.

23. Joensuu H. Gastrointestinal stromal tumor (GIST). Ann Oncol 2006; 17 (Suppl 10): 280-6.

24. Garrido MS, Moncada MM, Tapia GN, Méndez GO, Galindo HA, Huete AG et al. Efecto de un inhibidor tirosino kinasa (Imatinib) en pacientes con tumores estromales gastrointestinales metastásicos. Experiencia preliminar. Rev Méd Chile 2007; 135: 1327-32. 